\title{
Influence of WeChat on sleep quality among undergraduates in Chongqing, China: a cross-sectional study
}

\author{
Xianglong Xu1, ${ }^{1,2,3}$, Qianyi Lin ${ }^{4}$, Yan Zhang ${ }^{1,2,3}$, Runzhi Zhu ${ }^{1,2,3}$, Manoj Sharma ${ }^{5}$ and Yong Zhao ${ }^{1,2,3^{*}}$
}

\author{
*Correspondence: \\ zhaoyongzb@qq.com; \\ zhaoyong@cqmu.edu.cn \\ 1 School of Public Health \\ and Management, \\ Chongqing Medical \\ University, No. 1 Yixueyuan \\ Road, Yuzhong District, \\ Chongqing 400016, China \\ Full list of author information \\ is available at the end of the \\ article
}

\begin{abstract}
Background: Previous studies showed that social media is associated with sleep quality. WeChat (a native social media in China) is very popular in China, especially among the youth. In the second quarter of 2016, Tencent's WeChat had 806 million monthly active users. The study sought to identify the influence of WeChat on the sleep quality among undergraduate students.
\end{abstract}

Methods: A cross-sectional survey adopted a multi-stage stratified sampling survey to investigate undergraduates in Chongqing, China. Data were collected on 1979 eligible adults, aged 20.27 (SD: 1.26) years old, using Pittsburgh Sleep Quality Index (PSQI) to measure sleep quality.

Results: Respondents aged $20.27 \pm 1.26$ years included 535 (27.0\%) males, and 1311 (66.3\%) reported as having poor sleep quality. Of the 1979 participants, 1320 (66.70\%) were WeChat users. In multivariable analyses, gender, grade, nationality, living costs, the student leader, the only child, type of university, WeChat usage was associated with domains of PSQI among undergraduates ( $p<0.05$ for all). Compared with non-users, WeChat users had a lower score of subjective quality of sleep, sleep latency, use of sleeping medication, daytime dysfunction, and global PSQI score ( $p<0.05$ for all).

Conclusions: WeChat users may have better sleep quality than non-WeChat users among undergraduates. To determine causal relationships, further longitudinal studies will be required to test for the association between WeChat users and sleep quality. This study may also provide some implications for health promotion on sleep quality of undergraduate students.

Keywords: WeChat, Sleep quality, Undergraduates, Social media, China

\section{Background}

In recent years, China has witnessed an unprecedented boom in the use of social media. WeChat (Wikipedia 2011), first launched in January 2011, is a mobile communication tool developed by Tencent in China. As an instant message application, WeChat mainly focuses on the communication functions like sending text, voice, video and photo messages, group chat, scanning quick response code to add friends, and finding new friends nearby with advancement such as supporting moments (a built-in platform for sharing photos, texts, and links to friends), games, mobile payment, subscriptions of official accounts (providing service or contents by third-party individuals or organizations), and 
other assistant functions (Chen 2015). WeChat would include some new features compared to Whatsapp, a more popular social media in western countries. It has integrated video calling option which is not the case with Whatsapp. Differently from WeChat, it is possible for Skype users to make phone calls to land lines and cellphones around the world, at rates that are fixed and generally low. Facebook is now, by a very wide margin, which allows its users to create their own Facebook page, add friends and share personal updates and photos. WeChat is a type of social media that has gained considerable popularity among the young population in China. In the second quarter of 2016, Tencent's WeChat had 806 million monthly active users (The Statistics Portal 2016). There was a significant increase in frequency and long duration of WeChat use and $25 \%$ users checking WeChat more than 30 times per day, according to the user and platform data shared by Tencent in January 2015 (Tencent 2015).

Sleep problems and related daytime impairment are risk factors for numerous problems among college students including poor physical and mental health (Lund et al. 2010; Taylor et al. 2011), decrements in academic performance (Gaultney 2010), difficulty concentrating, and poorer lifestyle factors. Poor sleep quality among college students is also predictive of problem drinking (Kenney et al. 2012), and suicidal thoughts (Nadorff et al. 2011). In a 2012 American national survey of college students, 26.4\% reported sleep difficulties during the previous 12 months as being "traumatic or very difficult to handle", and $57.1 \%$ of the sample reported having enough sleep to feel rested on fewer than 4 days a week (ACHA 2012). Lack of sleep has been one of the most important public health problems in China and been neglected for a long time. Undergraduate students suffer from sleep problems around the globe (Lund et al. 2010), as well as in China more specifically (Suen et al. 2008). Most college students are sleep deprived, as $70.6 \%$ of students report obtaining $<8 \mathrm{~h}$ of sleep (Lund et al. 2010). A similar study among Chinese college students reported an average duration of sleep during weekdays to be $6.9 \mathrm{~h}$ (Tsui and Wing 2009). The prevalence of poor sleep quality was $54.8 \%$ among Chinese college student population in 2015 (Huang et al. 2015). In a Chinese study, 19\% of the medical students were found to have poor sleep quality as assessed by the Pittsburgh Sleep Quality Index (PSQI), with differences seen between years of study but not between genders (Feng et al. 2005).

Sleep issues may be associated with other student risk behaviors related to voluntary lifestyle choices including drinking patterns (Wechsler et al. 2000), sexual activities (Clemmens et al. 2004) and other lifestyle choices such as physical activity, and increasing use of technology, increased work and social demands (Chokroverty 2009; Trockel et al. 2000). Poor sleep quality was found to be associated with less life satisfaction, habit of delayed sleep and no breakfast, negative life events, perceived stress level and symptoms of depression (Lemma et al. 2012; Liu et al. 1995). Today's university students experience great psychological pressure due to the changing career market and increased competition for jobs (Biggeri et al. 2001). However, the timing and expression of sleep and wakefulness are highly influenced by environmental factors (Buysse et al. 2003). Although there might be significant variations from campus to campus, college culture is characterized by social arrangements and behavior practices that negatively affect students' sleep, including communal living, frequent parties and drinking, poor time management, and high levels of stress and work (Buboltz et al. 2002; Jensen 2003; Wechsler 
et al. 2000). Lifestyle, social and academic schedules, and insufficient sleep education could all contribute to the aetiology of chronic sleep insufficiency and poor sleep in university students (Brown et al. 2002, 2006; Buboltz et al. 2001).

Previous studies showed that social media is associated with sleep quality. A study in Flanders, Belgium showed that there was no safe dose and no safe time for using the mobile phone for text messaging or for calling after lights out (Van den Bulck 2007). A previous study has shown that the prevalence of Facebook dependence is associated with poor sleep quality (Wolniczak et al. 2013). Higher social media use volume and frequency had significantly greater odds of having sleep disturbance among young adults (Levenson et al. 2016). Smartphone overuse may lead to depression and/or anxiety, which in turn leads to sleep problems (Demirci et al. 2015). Excessive use of social media might result in misuse, dependence, and addiction (Griffiths 2012). A possible mechanism for the association of social media use and sleep quality is that the continuous light stimulation of an electronic screen could be sleep quality (Liu and Zhao 2014) as it altering the circadian rhythm, leading to insomnia and excessive sleepiness (Barion and Zee 2007). Moreover, a decrease in sleep duration leads to an increase in daytime sleepiness, influencing learning efficiency and academic performance, especially for undergraduates (Gomes et al. 2011).

However, no study has yet investigated the association between sleep quality and WeChat use among Chinese undergraduates. To our knowledge, the data regarding sleep patterns and habits in the Chinese undergraduate are limited. The effects of WeChat on the sleep quality of undergraduate students have not been studied thoroughly. Clarifying the effects of WeChat on the sleep quality of undergraduate students is necessary for the further exploration of the association between social media and sleeping problems particularly in China. The purpose of this study was to identify the relationship between WeChat use and sleep quality of undergraduate students, and increase the awareness of undergraduate about the importance of adopting healthy sleeping habits by addressing three research questions as follows: (1) What are the factors that affect WeChat usage among college students in China; (2) the effect of WeChat on sleep quality, (3) whether using WeChat has serious consequence on quality of sleeping.

\section{Methods}

\section{Study design}

The study design and methods, including the population and sample and the sampling framework, as well as the survey administration, pilot study and questionnaire development process have been reported previously (Xu et al. 2015). A cross-sectional study was conducted on undergraduates in Chongqing University Town. Chongqing University Town is located in Shapingba district, about $15 \mathrm{~km}$ from the downtown area of Chongqing. According to literature (Cheng et al. 2012), the prevalence of poor sleep quality was $54.7 \%$ of the total student population. We set $\mathrm{P}=0.547$. $(P=0.547 ; Q=1-P=1-0.547=0.453$ margin of error $d=0.1, P=0.1 \times 0.547=0.0547, Z_{\alpha}=1.96$; the sampling size is $N=\frac{Z_{\alpha}^{2} \times p q}{d^{2}}=\frac{1.96^{2} \times 0.547 \times 0.453}{0.0547^{2}}=318$; sampling size $=318$ ). The respondents were all students attending the selected classes. Respondents included 2100 volunteer undergraduate students, who were selected through probability proportionate sampling. 
Among the 2100 students, the response rate was 2088 (99.43\%). Nine responses were deleted because missing data, and thus the final sample comprised 1979 (94.24\%) respondents.

\section{Ethical approval}

The study was conducted in accordance with the Declaration of Helsinki, and the protocol was approved by the Ethics Committee of Chongqing Medical University (2013036). The protocol and procedures were explained verbally to each subject prior to obtaining written consent to participate. A pilot study was conducted in June 2014 to test the feasibility of the proposed study.

\section{Questionnaire}

The present study used the PSQI to assess sleep quality, which is widely accepted as both valid and reliable (Trockel et al. 2000) and has been previously used to assess undergraduate students in Taiwan (Kang and Chen 2009). The Cronbach alphas of global PSQI in this study was found to be 0.643 , the reliability is good. The collected information included:

1. Demographic information from each subject, such as type of university (Comprehensive University/University of Foreign Languages/Medical University/Normal University/Technological University), student leader, age, gender, grade, living expenses, and race; according to the Ministry of Education on the division of subjects and subjects' proportion in a university, universities can be divided into 13 different kinds (Wu 2002). University student leaders organize and coordinate common students to realize the goal of university education within the scope of duties and authority management (Shi 2008).

2. The questionnaire provided a screening instrument for using WeChat: 1 . Do you use WeChat more than once in the recent week? (yes/no)

3. PSQI; Component 1: subjective quality of sleep; Component 2: sleep latency; Component 3: sleep duration; Component 4: habitual sleep efficiency; Component 5: sleep disorders; Component 6: use of sleeping medication; Component 7: daytime dysfunction. The PSQI includes 19 items, and yields a score from 0 (good quality) to 21 (poor quality). Sleep onset latency and sleep efficiency, defined as the actual sleep time divided by the time in bed, are also obtained with the PSQI.

4. How social media affects sleep quality, including sleep onset latency, multiple arousals during sleep, waking up in the morning, memory, mood, fatigue at work, and feeling upset at daytime.

\section{Data analysis}

The characteristics of the participants were summarized using either means and standard deviations or frequencies and percentages. Chi square tests and $t$ test were used in the study. Multiple linear regression analyses were used to probe factors associated with domains of sleep quality among undergraduate students. Several factors were considered in modeling the factors that affect the sleep quality. These factors included sex (male/female), grade (freshman/sophomore/junior), student leader (yes/no), nationality 
(minority/han), only child (yes/no), in love (yes/no), living costs ( $<500 \mathrm{CNY} / 500-800$ $\mathrm{CNY} / 800-1000 \mathrm{CNY} /$ more than $1000 \mathrm{CNY}$ ), type of university (Comprehensive University/University of Foreign Languages/Medical University/Normal University/Technological University), and WeChat user (Yes/No). All statistics were performed using two-sided tests, and statistical significance was considered at $\mathrm{p}<0.05$. All data analyses were performed using a statistical software (SAS version 9.1; SAS Institute, Cary, NC, USA).

\section{Results}

Of the 1979 participants, 1320 (66.70\%) reported using WeChat. Significant differences between WeChat users and WeChat non-users were found in type of university (Comprehensive University: 6.6 vs. $33.4 \%$; Medical University: 61.9 vs. $38.1 \%$; Technological University: 66.2 vs. $33.9 \%$; Normal University: 65.0 vs. $35.0 \%$; University of Foreign Languages: 72.9 vs. $27.1 \%$ ), grade (Freshman: 31.6 vs. $41.9 \%$; Sophomore: 36.2 vs. $34.8 \%$; Junior: 32.1 vs. $23.4 \%$ ), student leaders ( 35.5 vs. $28.5 \%)$, courtship (32.7 vs. $27.2 \%$ ) and monthly living expense (<800 Yuan: 60.2 vs. $39.8 \%$; $800-1000$ Yuan: 72.7 vs. $27.3 \%$; more than 1000 Yuan: 76.8 vs. 23.2\%). Results are shown in Table 1.

Table 2 shows the multivariable analyses, which was used to predict the factors that influenced sleep quality. Compared with non-users, WeChat users had a lower score of subjective quality of sleep, sleep latency, use of sleeping medication, habitual sleep efficiency, daytime dysfunction, and global PSQI score. Compared with female, male had a lower score of subjective quality of sleep, sleep latency, habitual sleep efficiency, sleep disorders, daytime dysfunction and global PSQI score. However, females had a lower score of sleep duration than males. Age was not significantly associated with the domains of sleep quality. Compared with freshmen, sophomores had higher score of sleep duration. Compared with freshmen, sophomores had lower score of habitual sleep efficiency. Compared with freshmen, Junior had lower score of habitual sleep efficiency and global PSQI score. Respondents who with a living cost of $¥ 800-1000$ had higher score of habitual sleep efficiency than those with less than $¥ 800$. Compared with those was not the student leader, those were the student cadre had a lower score of global PSQI score. Compared with Han students, minority students had higher score of sleep duration but not the others. Compared with those were not the only child, those were the only child had a lower score of subjective quality of sleep, sleep latency, habitual sleep efficiency, sleep disorders, and global PSQI score. Compared with those were in love, those were not in love had higher score of habitual sleep efficiency. Compared with Comprehensive University, students from Medical University had a lower score of daytime dysfunction and habitual sleep efficiency. Compared with Comprehensive University, students from Technological University had higher score of sleep duration and had a lower score of daytime dysfunction and habitual sleep efficiency. Compared with Comprehensive University, students from Normal University had higher score of sleep duration and had a lower score of daytime dysfunction and habitual sleep efficiency. Compared with Comprehensive University, students from University of Foreign Languages had a lower score of daytime dysfunction, habitual sleep efficiency and global PSQI score. 
Table 1 The demographic characteristics of WeChat users and WeChat non users (n, \%)

\begin{tabular}{|c|c|c|c|}
\hline Item & WeChat users & WeChat non users & $\mathrm{p}$ \\
\hline Gender & & & $<0.001^{*}$ \\
\hline Male & $304(23.0 \%)$ & $231(35.1 \%)$ & \\
\hline Female & $1016(77.0 \%)$ & $428(65.0 \%)$ & \\
\hline Age (years) & $20.3 \pm 1.2$ & $20.2 \pm 1.3$ & 0.176 \\
\hline Grade & & & $<0.001^{*}$ \\
\hline Freshman & $417(31.6 \%)$ & $276(41.9 \%)$ & \\
\hline Sophomore & $479(36.2 \%)$ & $229(34.8 \%)$ & \\
\hline Junior & $424(32.1 \%)$ & $154(23.4 \%)$ & \\
\hline Whether the student leader & 0.002 & & \\
\hline Yes & $469(35.5 \%)$ & $188(28.5 \%)$ & \\
\hline No & $851(64.5 \%)$ & $471(71.5 \%)$ & \\
\hline Nationality & & & 0.327 \\
\hline Minority & $87(6.6 \%)$ & $36(5.5 \%)$ & \\
\hline Han & $1233(93.4 \%)$ & $623(94.5 \%)$ & \\
\hline The only child & 0.063 & & \\
\hline Yes & 705 (53.4\%) & $381(57.8 \%)$ & \\
\hline No & $615(46.6 \%)$ & $278(42.2 \%)$ & \\
\hline In love & & & 0.013 \\
\hline Yes & $431(32.7 \%)$ & $179(27.2 \%)$ & \\
\hline No & $889(67.4 \%)$ & $480(72.8 \%)$ & \\
\hline Living costs & & & $<0.001^{*}$ \\
\hline$<800$ & $298(60.2 \%)$ & $90(39.8 \%)$ & \\
\hline $800-1000$ & $647(72.7 \%)$ & $428(27.3 \%)$ & \\
\hline More than 1000 & $375(76.8 \%)$ & $141(23.2 \%)$ & \\
\hline Type of university & & & $0.012^{*}$ \\
\hline Comprehensive university & $261(66.6 \%)$ & $131(33.4 \%)$ & \\
\hline Medical university & $263(61.9 \%)$ & $162(38.1 \%)$ & \\
\hline Technological university & $258(66.2 \%)$ & $132(33.9 \%)$ & \\
\hline Normal university & $204(65.0 \%)$ & $110(35.0 \%)$ & \\
\hline University of foreign languages & $334(72.9 \%)$ & $124(27.1 \%)$ & \\
\hline
\end{tabular}

Chi square was used to compare differences in categorical variables, and t test was used to compare differences in continuous variables

* Statistically significant $(p<0.05)$

\section{Discussion}

This study found that WeChat users have better sleep quality in subjective quality of sleep, sleep latency, use of sleeping medication, daytime dysfunction, and global PSQI among undergraduates than non-WeChat users. Although higher social media use volume and frequency had significantly greater odds of having sleep disturbance among young adults (Levenson et al. 2016). Smartphone overuse may lead to depression and/ or anxiety, which in turn leads to sleep problems (Demirci et al. 2015). Similarly, Huber et al. (2002) reported that electromagnetic field exposure (mobile phone usage) in the evening influences physiological factors such as sleep quality and the melatonin rhythm, probably by influencing the brain activity. Maybe WeChat users have a reasonable time arrangement about WeChat and time spent on WeChat at daytime is more than that night especially before sleep. The use of WeChat has a limited effect on sleep. There are multiple environmental factors that affect their sleep-wake behavior, such as indulged 


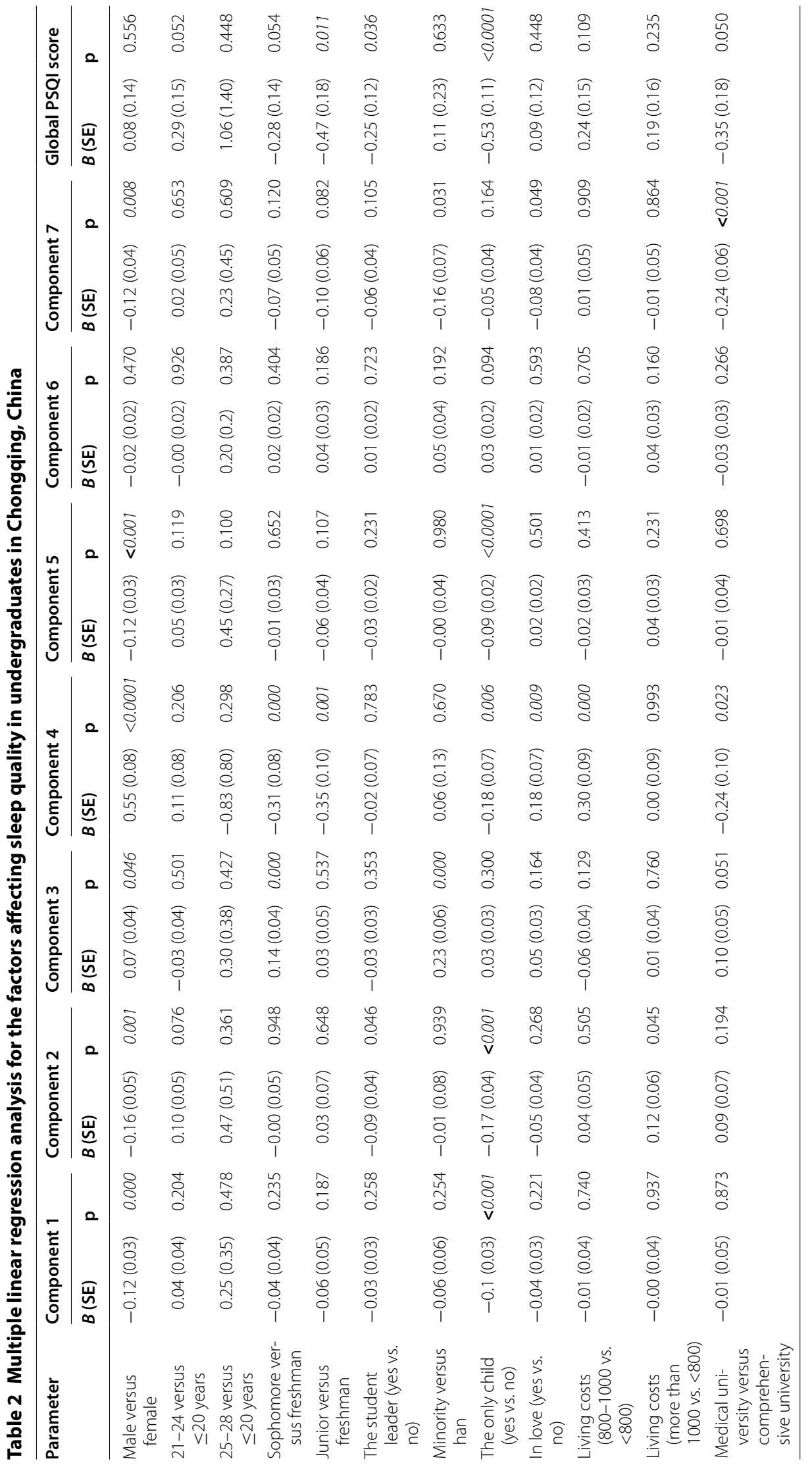




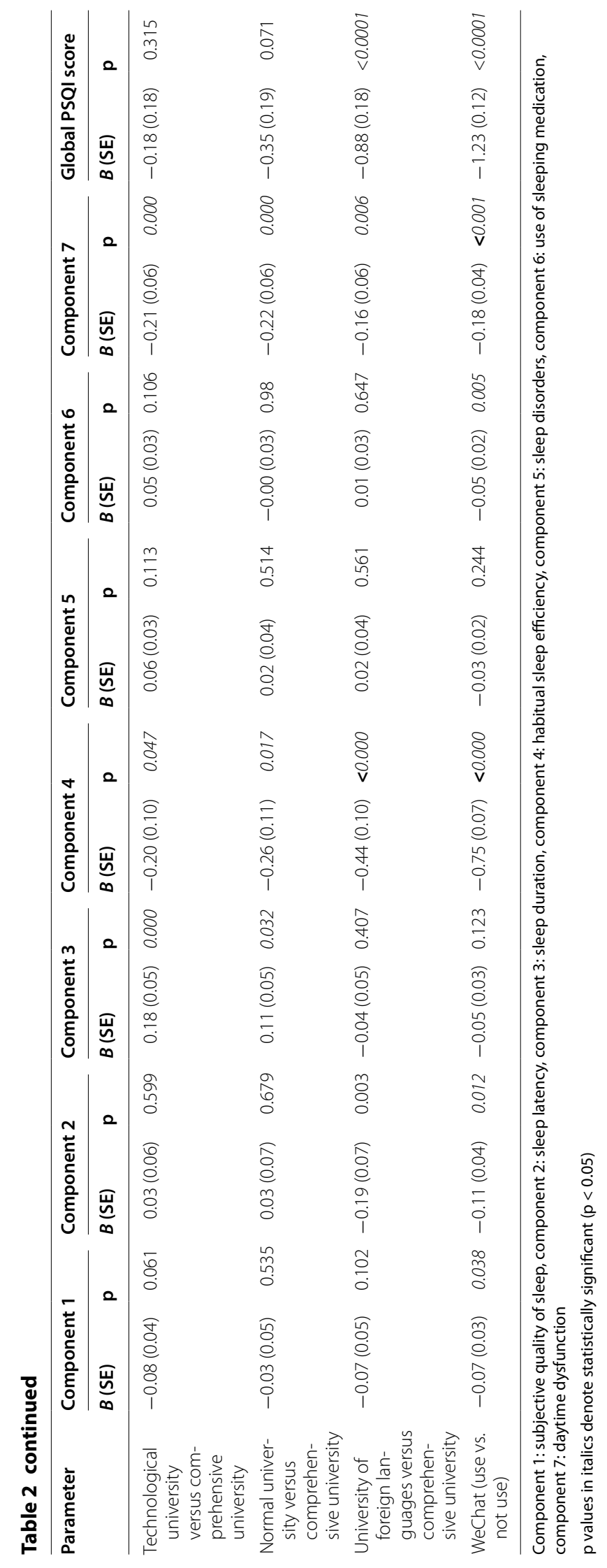


in extracurricular activities (Millman 2005). WeChat users spent more time on WeChat than non-users therefore reduced the opportunity that indulged in extracurricular activities. Sleep quality is a mediator between technology use after sleep onset and depression and anxiety in college students (Adams and Kisler 2013). Sleep loss more generally has been associated with stress, depression and increased anxiety (Chorney et al. 2008). One reason is that WeChat could be used to relieve stressful situations and feelings of depression or anxiety. WeChat users may have better sleep quality because of depression relieving. Furthermore, non-users may also use other social media; these may affect the quality of their sleep. Microblogging is now considered an indispensable part of a college student's life in China, and the excessive use of microblogs may bring about timemanagement problems (e.g., diminished quality and/or quantity of sleep time due to allday use of microblogs) (Hou et al. 2014). Another possible explanation for these findings may be that participants use WeChat may have access to more resources and information, including the benefits of good sleep, more education may help to improve their sleep quality. Sleep education programs can ensure that sleep knowledge and the level of salience allocated to sleep health are increased so that any poor sleep habits may be more of a choice than a lack of awareness (De Sousa et al. 2007). College age students are particularly susceptible to engaging in unhealthy sleep hygiene practices, and thus could benefit from greater awareness of the importance of good sleep (Forquer et al. 2008). For example, many WeChat Subscriptions (a kind of WeChat public platform) promote some health knowledge. However, this result does not mean that excessive use of WeChat could result in better sleep quality. Because we didn't incorporate every time spent on Wechat in our study, further research needs to be conducted to verify this result. It might help to increase the sleep quality and daytime functioning of these students. The study results suggest several other potential research avenues. For example, to acquire accurate the effect of WeChat on sleep quality among college students in general rather by institution, multi-site epidemiological cohort studies using validated screening tools and rigorous diagnostic criteria are needed.

This study also found that gender, grade, nationality, the student leader, the only child, living cost, type of university, in love were associated with domains of PSQI among undergraduates. Female college students may have more difficulty than male college students with sleep disturbances (Gaultney 2010). This is consistent with some other studies that found a significant association between gender and the poor sleep quality (Cheng et al. 2012). The third-year students did, however, have the lowest numerical mean global PSQI score and lowest prevalence of poor sleepers (Cates et al. 2015). Furthermore, short sleep appeared to be more common among minority race/ethnic groups, although the association was not significant (Whinnery et al. 2014). Student leader in University spent more time on work with high work demands and stress than common students. Barnes et al. (2012) found that time spent working is negatively associated with self-report sleep time. Children's sleep disturbances were associated with their home environment that may be influenced by caregivers' characteristics (Chen et al. 2014). The only child in the family usually got more attention than others about sleep health. Previous study found that economic stresses affect sleep-wake behavior of University students (Millman 2005). Although there might be significant variations from campus to campus, poor sleep quality and sleep deprivation were prevalent in university business 
students in Hong Kong, especially for those attending early morning lectures and living on-campus (Tsui and Wing 2009). In a Chinese study, 19\% of the medical students were found to have poor sleep quality as assessed by PSQI, with differences seen between years of study but not between genders (Feng et al. 2005). In terms of self-reported short sleep, divorced and never married individuals were more likely than married respondents to report short sleep (Whinnery et al. 2014).

The results found that students from University of Foreign Languages were less likely to have poor sleep quality. This study further confirmed that female students in the sample were more likely to report poor sleep quality in Chinese undergraduates (Petrov et al. 2014). Besides, the majority of undergraduates in University of Foreign Languages in Chongqing are females which may influence the final result. Although there might be significant variations from campus to campus, college culture is characterized by social arrangements and behavior practices that negatively affect students' sleep, including communal living, frequent parties and drinking, poor time management, and high levels of stress and work (Buboltz et al. 2002; Jensen 2003; Wechsler et al. 2000).

This study also has several limitations that need to be addressed. First, cross-sectional survey data reduced the ability of the study to make direct causal inferences, explore whether unmeasured factors can explain the observed relationships better, and determine the direction of causality. Second, this study did not conduct the psychometrics of the questionnaire. The future studies may include the psychometrics to assess sleep quality. Third, the sample is dominated by females. Fourth, we did have information about obesity that may confound the effect. Fifth, timing of use of WeChat is unknown and we did not evaluate the duration of WeChat in study. Future research is necessary to measure use of WeChat. Finally, the study was based on self-reports, which have their own limitations, as participants can be dishonest or not provide the correct responses. Notwithstanding the above limitations, this study also has certain strengths. This study is the first one to explore the effects of WeChat on the sleep quality of undergraduates in China. This exploratory study provides a workable framework for further exploration of the association between social media and sleeping problems, especially in China. This approach may help solve the increasingly serious sleeping problems of college students by providing a theoretical foundation for intervention.

\section{Conclusions}

This study found that WeChat users may have better sleep quality in subjective quality of sleep, sleep latency, use of sleeping medication, daytime dysfunction, and global PSQI among undergraduates than non-WeChat users. This study is the first study to investigate the influence of WeChat on the sleep quality of undergraduates in China and that provides a workable framework for further exploration of the association between social media and sleeping problems particularly in China. This study suggests that social media (e.g., WeChat) can be used for health promotion to improve sleep or health related behaviors (such as exercise and eating habits). This research study holds significance for researchers and practitioners in health education and health promotion as it might provide suggestion for building prevention programs. To determine causal relationships, further longitudinal studies will be required to test for the association between WeChat users and sleep quality. 


\section{Authors' contributions}

$X X$ was responsible for literature searches, the interpretation of the results, and writing the manuscript. XX and YZ participated in the development of the study protocol, data collection, and analysis. XX, QL, YZ, RZ, MS contributed to the drafting of the manuscript. YZ was responsible for the conception of the study and overall supervision of the data collection and analysis, the interpretation of the results, and manuscript preparation. All authors read and approved the final manuscript.

\section{Author details}

${ }^{1}$ School of Public Health and Management, Chongqing Medical University, No. 1 Yixueyuan Road, Yuzhong District, Chongqing 400016, China. ${ }^{2}$ Research Center for Medicine and Social Development, Chongqing Medical University, Chongqing 400016, China. ${ }^{3}$ Collaborative Innovation Center of Social Risks Governance in Health, Chongqing Medical University, Chongqing 400016, China. ${ }^{4}$ School of the Second Clinical, Chongqing Medical University, No. 1 Yixueyuan Road, Yuzhong District, Chongqing 400016, China. ${ }^{5}$ Department of Behavioral and Environmental Health, Jackson State University, Jackson, MS 39213, USA.

\section{Acknowledgements}

This study was funded by Pilot Study of Intervention to Improve Well-being of Residents by Microblog (Chongqing Social-scientific Plan Project (cultivation), Project No: 2013PYSH04), Summer Social Practice Project of School of Public Health and Management, Chongqing Medical University. The study sponsor had no role in study design. Thanks Wenjing Zhou, Qian Zhang, Xiaofang Yang, Yizhou Long, Jia Wang, Long Qin, Wenqi Xiong, Xingyan Li, Haixiao Chen in Chongqing Medical University for their active participation.

Competing interests

The authors declare that they have no competing interests.

Received: 2 March 2016 Accepted: 23 November 2016

Published online: 01 December 2016

\section{References}

Adams SK, Kisler TS (2013) Sleep quality as a mediator between technology-related sleep quality, depression, and anxiety. Cyberpsychol Behav Soc Netw 16(1):25-30

American College Health Association (2012) Reference group summary report spring 2012. http://www.acha-ncha.org/ docs/ACHA-NCHA-II_ReferenceGroup_DataReport_Spring2012.pdf. Accessed 4 June 2013

Barion A, Zee PC (2007) A clinical approach to circadian rhythm sleep disorders. Sleep Med 8(6):566-577

Barnes CM, Wagner DT, Ghumman S (2012) Borrowing from sleep to pay work and family Expanding time-based conflict to the broader non-work domain. Pers Psychol 65:789-819

Biggeri L, Bini L, Grilli L (2001) The transition from university to work: a multilevel approach to the analysis of the time to obtain the first job. J R Stat Soci Ser A (Stat Soc) 164(2):293-305

Brown FC, Buboltz WC, Soper B (2002) Relationship of sleep hygiene awareness, sleep hygiene practices, and sleep quality in university students. Behav Med 28:33-38

Brown FC, Buboltz WC, Soper B (2006) Development and evaluation of the sleep treatment and education program for students (STEPS). J Am Coll Health 54:231-237

Buboltz WC, Brown F, Soper B (2001) Sleep habits and patterns of college students: a preliminary study. J Am Coll Health 50:131-135

Buboltz WC Jr, Soper B, Brown F, Jenkins S (2002) Treatment approaches for sleep difficulties in college students. Couns Psychol Q 15:229-237

Buysse DJ, Barzansky B, Dinges D et al (2003) Sleep, fatigue, and medical training; setting an agenda for optimal learning and patients care. A report from the conference "Sleep, fatigue, and medical training: optimizing learning and the patient care environment". Sleep 2:218-225

Cates ME, Clark A, Woolley TW, Saunders A (2015) Sleep quality among pharmacy students. Am J Pharm Educ 79(1):09

Chen DD (2015) The influence of narcissism, leisure boredom, and gratifications on WeChat use and addiction. Chinese University of Hong Kong

Chen X, Gelaye B, Velez JC et al (2014) Attitudes, beliefs, and perceptions of caregivers and rehabilitation providers about disabled children's sleep health: a qualitative study. BMC Pediatr 14:245

Cheng SH, Shih CC, Lee IH, Hou YW, Chen KC, Chen KT, Yang YK, Yang YC (2012) A study on the sleep quality of incoming university students. Psychiatry Res 197(3):270-274

Chokroverty S (2009) Sleep disorders medicine basic science, technical considerations, and clinical aspects. Saunders, Philadelphia

Chorney DB, Detweiler MF, Morris TL, Kuhn BR (2008) The interplay of sleep disturbance, anxiety, and depression in children. J Pediatr Psychol 33(4):339-348

Clemmens D, Engler A, Chinn PL (2004) Learning and living health: college students' experiences with an introductory health course. J Nurs Educ 43(7):313

De Sousa IC, Araujo JF, De Azevedo CVM (2007) The effect of a sleep hygiene education program on the sleep-wake cycle of Brazilian adolescent students. Sleep Biol Rhythms 5:251-258

Demirci K, Akgönül M, Akpinar A (2015) Relationship of smartphone use severity with sleep quality, depression, and anxiety in university students. J Behav Addict 4(2):85-92

Feng G, Chen J, Yang X (2005) Study on the status and quality of sleep-related influencing factors in medical college students. Chin J Epidemiol 26:328-331 (In Chinese) 
Forquer LM, Camden AE, Gabriau KM, Johnson CM (2008) Sleep patterns of college students at a public university. J Am Coll Health 56(5):563-565

Gaultney JF (2010) The prevalence of sleep disorders in college students: impact on academic performance. J Am Coll Health 59(2):91-97

Gomes AA, Tavares J, de Azevedo MH (2011) Sleep and academic performance in undergraduates: a multi-measure, multi-predictor approach. Chronobiol Int 28(9):786-801

Griffiths MD (2012) Facebook addiction: concerns, criticism, and recommendations-a response to Andreassen and colleagues. Psychol Rep 110(2):518-520

Hou J, Huang Z, Li H et al (2014) Is the excessive use of microblogs an internet addiction? Developing a scale for assessing the excessive use of microblogs in Chinese college students. PLoS One 9(11):e110960

Huang ZG, Shan ZG, Yang YH (2015) Study on college students' sleep status and related factors. In: 4th national statistical modeling contest for college students in China. http://cpfd.cnki.com.cn/Article/CPFDTOTAL-GTJX201511001019. htm. Accessed 29 Nov 2015 (in Chinese)

Huber R, Treyer V, Borbély AA, Schuderer J, Gottselig JM, Landolt HP, Werth E, Berthold T, Kuster N, Buck A, Achermann PJ (2002) Electromagnetic fields, such as those from mobile phones, alter regional cerebral blood flow and sleep and waking EEG. Sleep Res 11(4):289-295

Jensen DR (2003) Understanding sleep disorders in a college student population. J Coll Couns 6:25-34

Kang J-H, Chen S-C (2009) Effects of an irregular bedtime schedule on sleep quality, daytime sleepiness, and fatigue among university students in Taiwan. BMC Public Health 9:248

Kenney SR, LaBrie JW, Hummer JF, Pham AT (2012) Global sleep quality as a moderator of alcohol consumption and consequences in college students. Addict Behav 37(4):507-512

Lemma S, Gelaye B, Berhane Y et al (2012) Sleep quality and its psychological correlates among university students in Ethiopia: a cross-sectional study. BMC Psychiatry 12:237

Levenson JC, Shensa A, Sidani JE, Colditz JB, Primack BA (2016) The association between social media use and sleep disturbance among young adults. Prev Med 85:36-41

Liu Z, Zhao Z (2014) Effects of light interruption on sleep and viability of Drosophila melanogaster. PLoS One 9:e105678

Liu X et al (1995) Sleep quality and its correlates in college students. Chin Ment Health J. http://en.cnki.com.cn/Article_ en/CJFDTOTAL-ZXWS504.001.htm. Accessed Apr 1995 (in Chinese)

Lund HG, Reider BD, Whiting AB, Prichard JR (2010) Sleep patterns and predictors of disturbed sleep in a large population of college students. J Adolesc Health 46(2):124-132

Millman RP, Working Group on Sleepiness in Adolescents/Young Adults, AAP Committee on Adolescence (2005) Excessive sleepiness in adolescents and young adults: causes, consequences, and treatment strategies. Pediatrics 115(6):1774-1786

Nadorff MR, Nazem S, Fiske A (2011) Insomnia symptoms, nightmares, and suicidal ideation in a college student sample. Sleep 34(1):93-98

Petrov ME, Lichstein KL, Baldwin CM (2014) Prevalence of sleep disorders by sex and ethnicity among older adolescents and emerging adults: relations to daytime functioning, working memory and mental health. J Adolesc 37(5):587-597

Shi G (2008) Theoretical thinking on the work of college student leaders. Stud Ideol Educ 1. http://www.cnki.com.cn/ Article/CJFDTOTAL-SIXI200801019.htm (in Chinese)

Suen LK, Hon KL, Tam WW (2008) Association between sleep behavior and sleep-related factors among university students in Hong Kong. Chronobiol Int 25(5):760-775

Taylor DJ, Gardner CE, Bramoweth AD, Williams JM, Roane BM, Grieser EA et al (2011) Insomnia and mental health in college students. Behav Sleep Med 9(2):107-116

Tencent (2015) The first data analysis report on WeChat platform. http://tech.qq.com/a/20150127/018482.htm\#p=1. Accessed 27 Jan 2015

The Statistics Portal (2016) Number of monthly active WeChat users from 2nd quarter 2010 to 2nd quarter 2016 (in millions). http://www.statista.com/statistics/255778/number-of-active-wechat-messenger-accounts/. Accessed Aug 2016

Trockel MT, Barnes MD, Egget DL (2000) Health-related variables and academic performance among first-year college students: implications for sleep and other behaviors. J Am Coll Health 49:125-131

Tsui YY, Wing YK (2009) A study on the sleep patterns and problems of university business students in Hong Kong. J Am Coll Health 58(2):167-176

Van den Bulck J (2007) Adolescent use of mobile phones for calling and for sending text messages after lights out: results from a prospective cohort study with a one-year follow-up. Sleep 30(9):1220-1223

WeChat Wikipedia (2011) https://en.wikipedia.org/wiki/WeChat. Accessed 21 Sep 2011

Wechsler H, Kelley K, Weitzman ER, San Giovanni JP, Seibring M (2000) What colleges are doing about student binge drinking: a survey of college administrators. J Am Coll Health 48:219-226

Whinnery J, Jackson N, Rattanaumpawan P, Grandner M (2014) Short and long sleep duration associated with race/ ethnicity, sociodemographics, and socioeconomic position. Sleep 37(3):601-611

Wolniczak I, Mego-Aquije K, Bernabe-Ortiz A et al (2013) Association between Facebook dependence and poor sleep quality: a study in a sample of undergraduate students in Peru. PLoS One 8(3):e59087

Wu S (2002) Re-discuss classification of Chinese university. China High Educ Eval 4 http://www.cnki.com.cn/Article/ CJFDTOTAL-GJPG200204013.htm (in Chinese)

Xu XL, Zhu RZ, Sharma M et al (2015) The influence of social media on sleep quality: a study of undergraduate students in Chongqing, China. J Nurs Care 4:253. doi:10.4172/2167-1168.1000253 